\title{
Study of seed hair growth in Populus tomentosa, an important character of female floral bud development
}

\author{
Meixia Ye ${ }^{1}$, Zhong Chen ${ }^{1 \dagger}$, Xiaoxing $\mathrm{Su}^{2}$, Lexiang $\mathrm{Ji}^{1}$, Jia Wang ${ }^{1}$, Weihua Liao ${ }^{1}$, Huandi Ma ${ }^{1}$ and Xinmin $\mathrm{An}^{1 *}$
}

\begin{abstract}
Background: Poplar seed hair is an environmental annoyance in northern China due to its abundance and widespread airborne distribution after maturation. The morphogenesis and molecular mechanisms of its development are not well understood, and little attention has been focused on the dynamics of its development. To better understand the mechanism of poplar seed hair development, paraffin sections were used to examine the initiation and elongation of poplar seed hairs. RNA-seq technology was also employed to provide a comprehensive overview of transcriptional changes that occur during seed hair development.
\end{abstract}

Results: The placenta at the base of ovary, was identified as the origin of seed hair development, which is in sharp contrast to cotton fibers that originate from epidermal cells of the seed coat. An enlarged cell nucleus in seed hair cells was also observed, which was supported by our gene ontology enrichment analysis. The significant enriched GO term of "endoreduplication" indicated that cycles of endoreduplication, bypassing normal mitosis, is the underlying mechanisms for the maintenance of the uni-cellular structure of seed hairs. By analyzing global changes in the transcriptome, many genes regulating cell cycle, cell elongation, cell well modification were identified. Additionally, in an analysis of differential expression, cellulose synthesis and cell wall biosynthesis-related biological processes were enriched, indicating that this component of fiber structure in poplar seed hairs is consistent with what is found in cotton fibers. Differentially expressed transcription factors exhibited a stage-specific up-regulation. A dramatic down-regulation was also revealed during the mid-to-late stage of poplar seed hair development, which may point to novel mechanisms regulating cell fate determination and cell elongation.

Conclusions: This study revealed the initiation site of poplar seed hairs and also provided a comprehensive overview of transcriptome dynamics during the process of seed hair development. The high level of resolution on dynamic changes in the transcriptome provided in this study may serve as a valuable resource for developing a more complete understanding of this important biological process.

Keywords: Seed hairs, Trichomes, Fiber, Poplar, Transcriptome

\section{Background}

Trichomes, an important feature used in taxonomy, are a common feature on many plants, appearing on the surface of leaves, petals, stems, petioles and peduncles. They play significant roles in protecting plants from insect feeding,

\footnotetext{
* Correspondence: anxinmin@bjfu.edu.cn

${ }^{\dagger}$ Equal contributors

${ }^{1}$ National Engineering Laboratory for Tree Breeding, Key Laboratory of Genetics and Breeding in Forest Trees and Ornamental Plants, The Tree and Ornamental Plant Breeding and Biotechnology Laboratory, Beijing Forestry University, Beijing 100083, People's Republic of China

Full list of author information is available at the end of the article
}

regulating temperature, decreasing water loss, and reducing mechanical abrasion [1-4]. In Arabidopsis, leaf epidermal trichomes have been extensively used to study trichome development [5,6]. For both Arabidopsis trichome cell and cotton fiber cell, two distinct stages (cell fate determination and cellular specification), that function as developmental 'switches', have been identified [6-8]. In recent years, many key genes determining trichome cell fate have been identified in trichome-related mutants. These include the MYB/bHLH/WD-repeat trichome-promoting complex comprised of the R2R3 MYB transcription factor, GLABROUS1(GL1), bHLH factors, GLABROUS3(GL3) 
and ENHANCER OF GLABRA3(EGL3), and a WD40repeat factor, TRANSPARENT TESTA GLABRA1(TTG1), which induces the expression of GLABRA2(GL2) and TTG2 [9-13]. TRICHOMELESS (TCL) and TRYPTY$\mathrm{CHON}$ (TRY), proteins that act as negative regulators, can move to neighbouring cells and compete with GL1 for binding to GL3/EGL3, blocking the formation of the trichome promoting complex, thereby rendering them as spacing or pavement cells. $[14,15]$. In addition to their value in cell fate research, trichomes, because of their single-celled structure, are also ideal for studying cell elongation, expansion, and developmental regulation. Another specialized type of trichome is commonly seen on the outside of seeds that facilitates seed dispersion over long distances, which includes cotton fibers, a seed trichome derived from individual cells of the epidermal layer of the seed coat. Compared to Arabidopsis trichomes, however, cotton fibers have many unique attributes. Their extremely large size and elongated structure have made them an ideal model for cell research. Additionally, cotton fibers are composed of nearly pure cellulose which had made them an optimal model for cellulose and cell wall biogenesis research.

Chinese white poplar (Populus tomentosa Carr.), a native tree species that plays an important role in forest production and urban green space in large areas of northern China, produces seed trichomes, which are commonly referred to as seed hairs. Poplar seed trichomes greatly facilitate the ability of seeds to float in the air and as a result, enhance their potential for long distance distribution by wind. When seed maturation occurs on a poplar catkin of an adult tree, the seed capsule dehisces and copious amounts of seed hair are released. The annual release of the seed hair has developed into a serious environmental annoyance, creating an extra urban health problem, especially in densely populated areas. Although poplar wood quality and the reproductive biology of poplar have been the focus of breeding research [16-21], little is known about the development of seed hairs. Therefore, it is essential to better understand how the initiation of poplar seed hairs is regulated at molecular level in order to inhibit or eliminate their formation using biotechnology.

Fortunately, recent advances in RNA-seq technology have increased its potential in generating functional "omics data" and thus help in elucidating the molecular basis for key developmental processes. In the current study, we examined the morphogenesis of poplar seed hairs by sectioning paraffin-embedded tissues and conducted a comprehensive overview of gene expression throughout seed hair development using deep sequencing technology. Combining the data from the two approaches has enabled a greater association between molecular-level data and the morphological changes in seed hair development in poplar.

\section{Results}

\section{Rapid morphological changes during development of} seed hairs

Floral buds and the later catkin samples were harvested at $0 \mathrm{~h}, 24 \mathrm{~h}, 34 \mathrm{~h}, 48 \mathrm{~h}, 58 \mathrm{~h}, 72 \mathrm{~h}, 96 \mathrm{~h}, 120 \mathrm{~h}$ from water-cultured cut branches. Ovaries embedded in paraffin were serially-sectioned to study seed hair morphogenesis. Observations were made of samples beginning at $0 \mathrm{~h}$ in order to get an overview of ovary structure. A complete ovary, comprised of two anatropous ovules with basal placentation and highly developed funicles (Figure 1A, B, C, D) could be observed, and the epidermal cells of the funicle were identified as origin of poplar seed hairs (Figure 1E, I). Staining of nuclei with saffranin was clearly evident in epidermal cells of the funicle in samples collected during the first four collection times (Figure 1I). These cells could easily be distinguished from other cells. The appearance of a substantial number of fibrous structures was unambiguous at $58 \mathrm{~h}$ (Figure 1J, K) and completely filled the ovary at $120 \mathrm{~h}$ (Figure 1H, L). Cells with deeply dyed nuclei were observed continuously during the rapid development of seed hairs but without any evidence of cell division (Figure 1I, J, K, L), thus resulting in a unicellular and unbranched seed hair trichome. Based on these results, three stages of seed hair morphogenesis were observed: initiation, elongation, and maturation.

\section{Sequence assembly and functional annotation}

mRNA from floral bud or the later catkin tissue of $P$. tomentosa at above-mentioned eight distinct development stage were extracted. After quality assessment, eight cDNA libraries were constructed and were sequenced by Illumina deep-sequencing platform. In order to preclude DNA contamination from other organisms, 500,000 randomly-sampled reads were aligned with the $\mathrm{Nt}$ database. Results indicated that the top ten species with the greatest similarity were all Populus, indicating that DNA contamination by other species was low, and that $P$. tomentosa had a high degree of homology with other poplar species. After removal of low-quality reads, a total of 150 million reads were available for transcript assembly, reflecting 115.006 gigabases which is equivalent to $\sim 35$ fold coverage of the $P$. trichocarpa genome. Inchworm step in this software extracted all overlapping $k$-mer from valid RNA-seq reads, then a greedy extension algorithm was applied to generate contigs based on the unique $(k-1)$-mer overlaps. Steps of Chrysalis and Butterfly generated all component and reported full-length transcripts for alternatively spliced isoforms. A mixed reads pool from all samples generated 344,412 transcripts greater than $100 \mathrm{bp}$ in length, and 213,096 unigenes, with an N50 of 1,721 bp and 754 bp for transcripts and unigenes, respectively. Length distribution of all assembled transcripts is presented in Figure 2. 

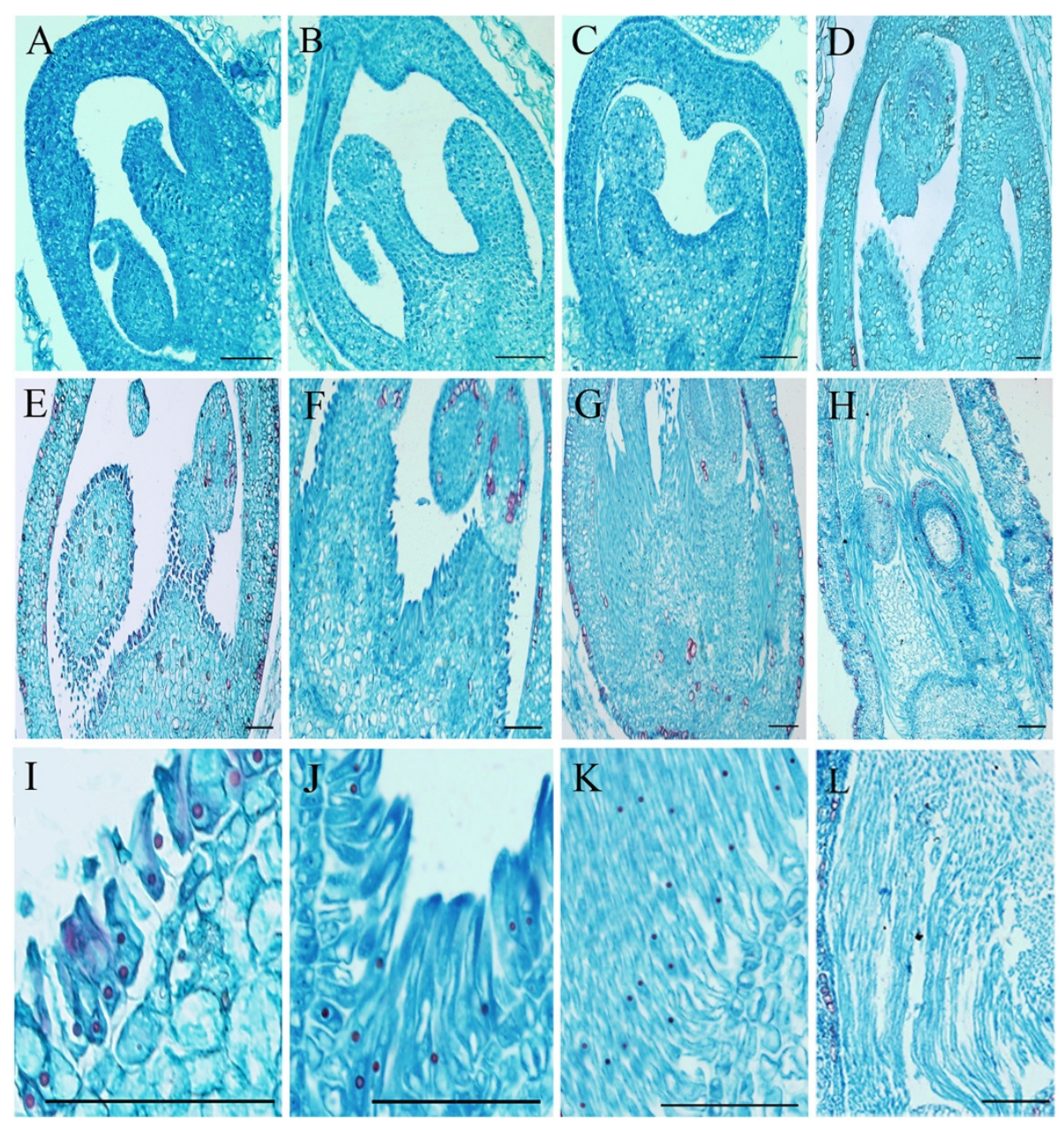

Figure 1 Digital images of ovary sections during poplar seed hair development. A, B, C, D represent observations made in samples collected at 0 h, 24 h, 34 h, 48 h, respectively No obvious trichome structures could be seen during this phase. E, F, G, H are samples observed at 58 h, 72 h, 96 h, $120 \mathrm{~h}$, respectively. Fiber initiation was observed in samples collected at $58 \mathrm{~h}$, after which fiber cells elongated from the placenta of the ovule. At $120 \mathrm{~h}$, the ovary was fully filled with fibers. I, J, K, L are higher magnifications of E, F, G, H, respectively. Bars in each photo represent $100 \mu \mathrm{m}$.

A BLASTx comparison showed that $24.30 \%$ of all unigenes shared a high similarity with proteins in the $\mathrm{Nr}$ database. Of these, $87 \%$ of sequences had the highest similarity to $P$. trichocarpa, while the remaining unigene sequences had a best match to Ricinus communis (4\%) and Vitis vinifera (3\%). The reason for the lack of an annotation for the remaining $75.3 \%$ of unigenes was the large proportion of short sequences. The higher percentage of unigene annotation hits found in our large-scale transcript sequences was due the availability of long sequence, e.g., $75.6 \%$ for transcripts above $300 \mathrm{bp}$, increasing the ability of aligning the sequence to known gene sequences.

To further understand the transcriptome library and to characterize biological processes associated with seed hair development, a gene ontology (GO) assignment was used to classify the function of each assembled sequence. Among all 213,096 unigenes, 37,892 were successfully annotated with hierarchical GO terms involving biological process, molecular function and cellular component. All leading GO terms at level 2 can be categorized into 63 groups (Figure 3 ). In addition to 'metabolic process' and 'cellular process' that contributed the most to biological processes, a large proportion of genes were assigned to the 'biological regulation', 'establishment of localization', and 'response to stimulus'. Significantly, in the category of molecular function, 'binding' was prominent, which suggested an important regulatory role for transcription factors during seed hair development. Another component 'catalytic activity' may also indicate significant metabolic activity. Genes identified with 'transporter activity' and 'structural molecule activity' may help to identify genes that play an important role in trichome component synthesis during seed hair formation and elongation.

A further comparison of all unigenes with the KEGG database identified several important pathways. 

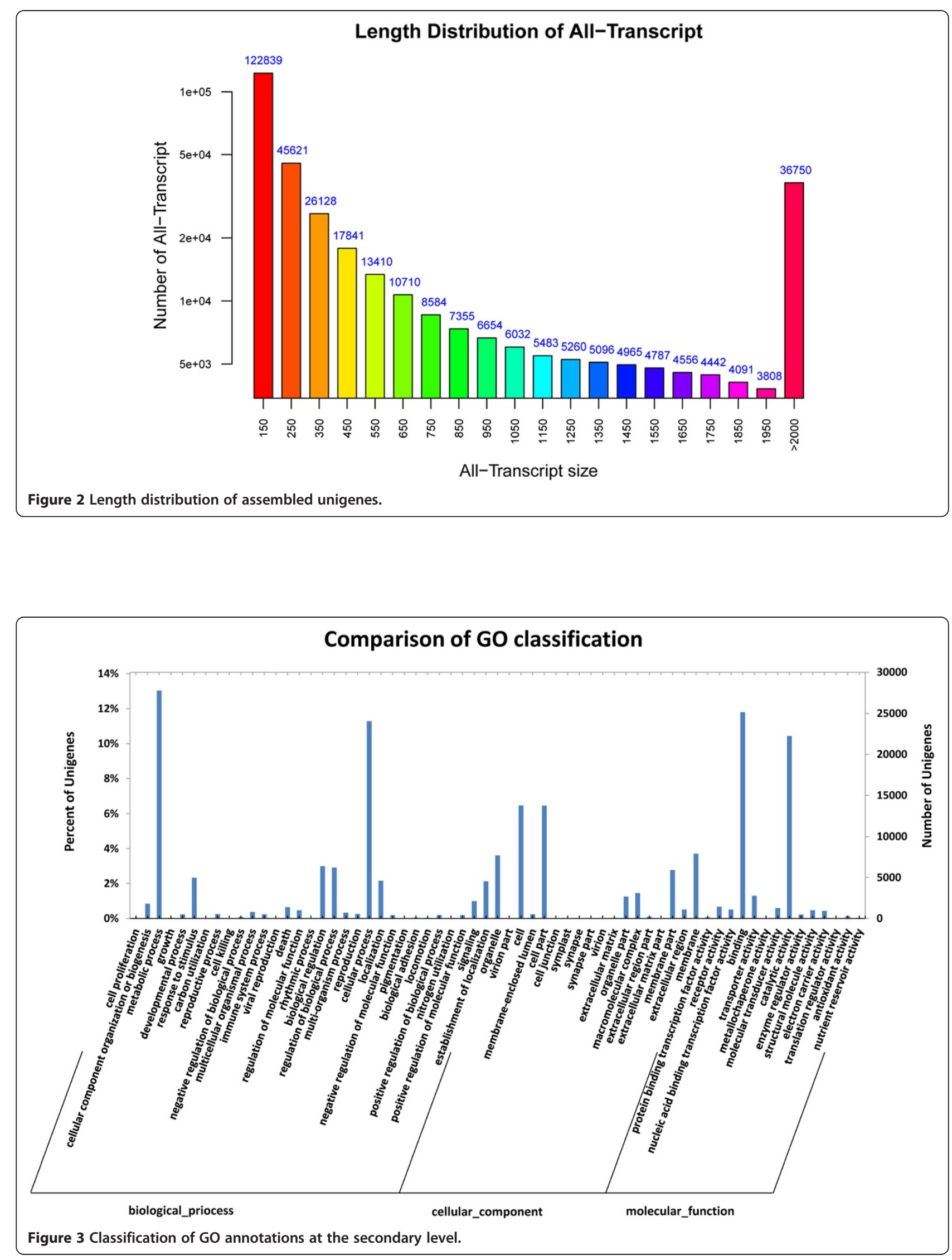
The represented pathways with the most annotated unigenes were 'ribosome' (ko03010), 'protein processing in endoplasmic reticulum' (ko04141), 'starch and sucrose metabolism' (ko00500), 'oxidative phosphorylation' (ko00190), 'glycolysis/gluconeogenesis' (ko00010) and 'plant hormone signal transduction' (ko04075). These associations greatly contribute to our understanding of the important processes and pathways involved in seed hair development.

\section{Changes in transcriptome profiles during seed hair development}

A total of 4,245 differentially expressed genes (DEGs) were identified during the course of seed hair development. Details on the number of DEGs at each time point are presented in Table 1. GO enrichment analysis was conducted for each two consecutive time points to better understand the functional role of the DEG. All significant GO terms with a criterion of $\mathrm{p}<0.05$ are listed in Additional file 1: Table S1. GO terms such as "cell wall modification", "cell wall macromolecule catabolic process", "cellular cell wall organization", "cell wall biogenesis", "structural constituent of cell wall" were all significantly enriched during the whole process of seed hair development. Additionally, "cellulose synthase activity", "1,4-beta-xylosidase activity", "cellulose biosynthetic process", "cellulose activity", and "endo-1,4-beta-xylanase activity" all of which are associated with fiber biogenesis were also found to be significantly enriched during the seed hair development. The cellulose-related results were consistent with the enrichment result of the abovementioned cell-wall-related GO terms, as cellulose is a main structural constituent of fiber cell walls.

Interestingly, in addition to cell wall and cellulose related enrichment, "regulation of cell division" and "regulation of cell shape" were also found to be significantly enriched at all time points. "DNA replication" related genes were significantly up-regulated, and the more specific terms belonging to "DNA replication" such as, "de novo pyrimidine base biosynthetic process", "DNA-dependent DNA replication" and "DNA unwinding involved in replication" were

Table 1 Number of differentially expressed transcripts at each sampling time point

\begin{tabular}{lll}
\hline Comparisons & $\begin{array}{l}\text { Number of up-regulated } \\
\text { transcripts }\end{array}$ & $\begin{array}{l}\text { Number of down- } \\
\text { regulated transcripts }\end{array}$ \\
\hline $24 \mathrm{~h}$ vs $0 \mathrm{~h}$ & 390 & 507 \\
$34 \mathrm{~h}$ vs $24 \mathrm{~h}$ & 164 & 70 \\
$48 \mathrm{~h}$ vs $34 \mathrm{~h}$ & 141 & 457 \\
$58 \mathrm{~h}$ vs $48 \mathrm{~h}$ & 368 & 300 \\
$72 \mathrm{~h}$ vs $58 \mathrm{~h}$ & 208 & 335 \\
$96 \mathrm{~h}$ vs $72 \mathrm{~h}$ & 220 & 64 \\
$120 \mathrm{~h}$ vs $96 \mathrm{~h}$ & 384 & 574 \\
\hline
\end{tabular}

still observed to be significantly enriched at $72 \mathrm{~h}$. Noticeably, significant enrichment of genes relating to "G1/S transition of mitotic cell cycle" and "exit from mitosis" were observed at $120 \mathrm{~h}$. Enrichment analysis for these last two time points indicated that the cell cycle may have been arrested, and maintained at a certain stage for a prolonged period of time. The time course used in this study covered the complete process of seed hair development from the view of anatomical morphology, as at $120 \mathrm{~h}$ seed hairs had entirely filled the ovary.

\section{Cell wall-related and cell elongation genes}

Since the analysis of differential expression identified a significant enrichment of cell wall and cellulose related GO terms, the expression of genes related to cellulose biogenesis was examined. Cellulose synthase genes, a class of genes involved in the synthesis of cellulose, exhibited elevated levels of expression during the mid to late period of the sampled time course (Figure 4A). Sucrose synthesis genes are another class of genes important to cellulose biogenesis because sucrose provides a substrate for cellulose synthesis and sucrose synthase catalyzes the synthesis of UDP-glucose. In general, genes encoding sucrose synthases exhibited a pattern of abundance (Figure 4B) similar to cellulose synthase genes, however, a small subset of them were also highly expressed at an early stage of seed hair development implying an association with trichome initiation. All of these results support the GO enrichment result of cell wall and cellulose related terms. Many other cell wall related genes were also identified including, pectinesterase, polygalacturonase, chitinase, xylanase and callose synthase genes, all of which are involved in cell wall modifications. Additionally, 19 expansin family genes, 3 unigenes encoding germin-like proteins, and 7 snakin unigenes were found. Expansin-A15, expansin-A8 and expansin-A1 exhibited a pattern of differential expression, with a sharp peak of expression in the mid-to-late stages of seed hair development.

Cell elongation is an important process in fiber development. "Ethylene biosynthetic process" was identified as one of the most significant enrichment terms in the GO analysis of DEGs at $72 \mathrm{~h}$ which represented a phase of development when seed hairs are highly elongated. A dramatic up-regulation of ACC oxidase during this phase $(72 \mathrm{~h})$ was also detected, indicating an association with seed hair trichome elongation. Many fiber elongation-related genes, homologous to those identified in cotton, were found in the present study. The majority of these genes exhibited a differential gene expression pattern (Figure $4 \mathrm{C}$ ), suggesting a role in trichome elongation in poplar. Among these genes, genes encoding E6 protein, 3-ketoacyl CoA synthase 6, EEF1A, and FIDDLEHEAD exhibited a much higher abundance than the other genes. 


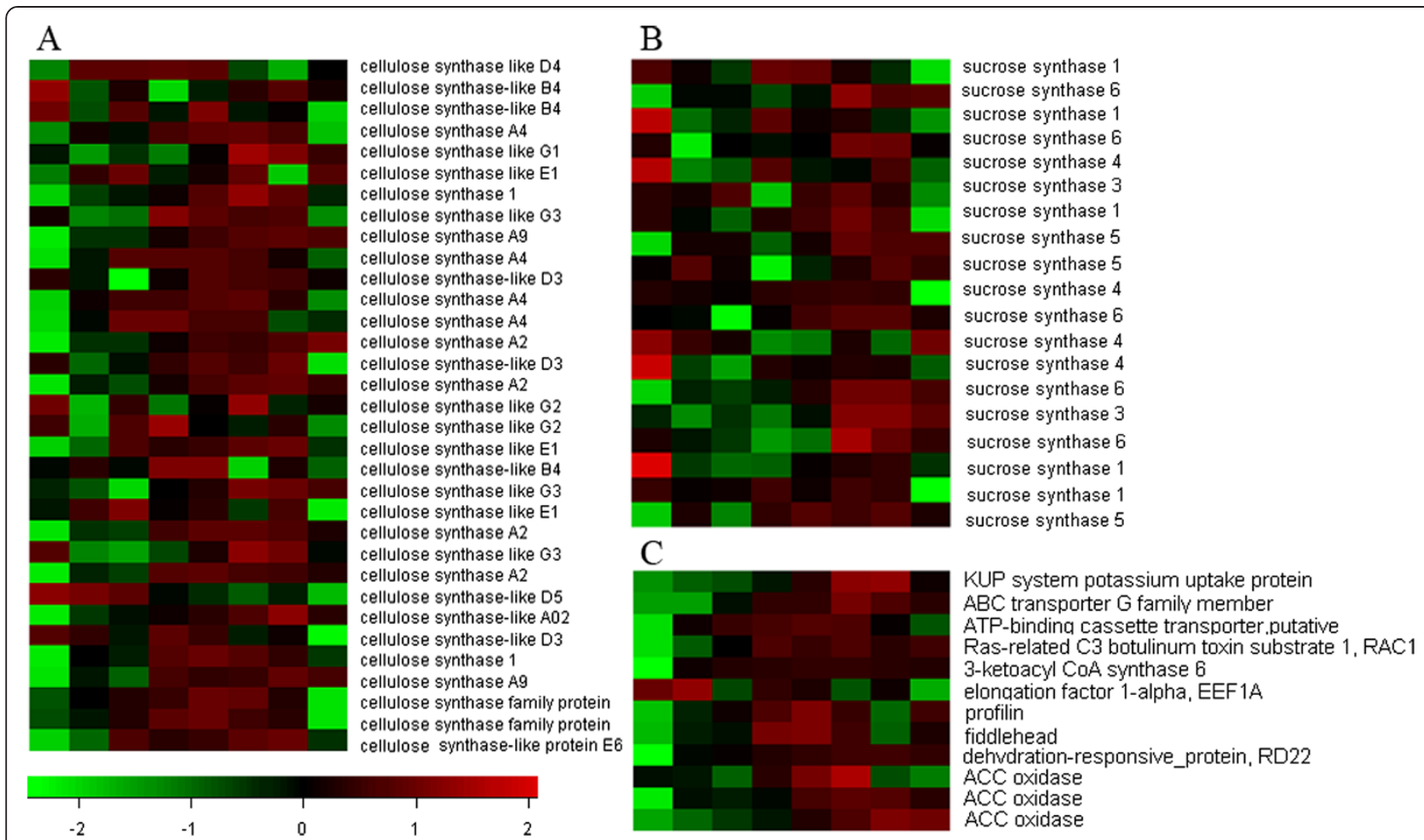

Figure 4 Heat map of gene expression. A, genes encoding cellulose synthases. B, genes encoding sucrose synthases. C, cell elongation related genes.

\section{Expression pattern of transcription factors}

Transcription factors, proteins that bind to specific motifs in the promoter of target genes, regulate gene expression. By comparing sequences obtained in this study to the Populus trichocarpa Transcription Factor database, 57 families of transcription factors were identified, covering nearly all families of transcription factors. NAC, bHLH, MYB, C2H2, B3, C3H, ERF, GRAS, WRKY and MYB-related families were the transcription factor families most represented in our study with 459, 290, 284, 259, 248, 220, 211, 205, 168 and 165 unigenes, respectively. After clustering the expression patterns of transcription factors that were differentially and highly expressed, several stage-specific expression patterns were identified. Three patterns of up-regulation were found in trichome developing stage, advanced trichome elongation stage, and the trichome-cell-determining stage (Figure 5). A pattern of down-regulation was also identified that occurred over the whole time course, e.g., the AP2 family.

Members within some certain transcription factor families, however, were not restricted to a specific expression pattern, as exemplified in YABBY, FAR1, bHLH, C2H2, C3H, ERF, GRF, MYKC, ZF-HD. These data suggest that they play an important role throughout seed hair development in poplar. Noticeably, GL2, a member of the HD-
ZIP transcription factor family, exhibited a high level of expression throughout the entire time course, with an upregulation at $24 \mathrm{~h}$ and $48 \mathrm{~h}$, which was consistent with its potential role in trichome cell fate determination. While other transcription factors associated with the formation of trichome-promoting complex exhibited a steady expression state, three of the most highly expressed $\mathrm{C} 2 \mathrm{H} 2$ unigenes significantly increased their expression over the sampling period, which suggested that they play a role in trichome development.

\section{Validation by real-time quantitative RT-qPCR}

To validate the reliability of RNA-seq data obtained in the current study, a total of 10 genes belonging to the ko00500 pathway (starch and sucrose metabolism) or the ko04075 pathway (plant hormone signal transduction) and the YABBY gene family were selected for real-time reverse transcription, quantitative PCR (RT-qPCR) analysis at all eight time points. The selected genes included genes encoding sucrose-phosphate synthase, trehalose 6-phosphatase, betaD-xylosidase 4, glucose-1-phosphate adenylyltransferase, polygalacturonase, beta-fructofuranosidase, protein phosphatase $2 \mathrm{C}-1 / \mathrm{PP} 2 \mathrm{C}-1$, protein phosphatase $2 \mathrm{C}-2 / \mathrm{PP} 2 \mathrm{C}-2$, YABBY-1, YABBY-2. Genes in these two pathways were selected because they were the top two pathways identified in this study and contained a large proportion of the identified 


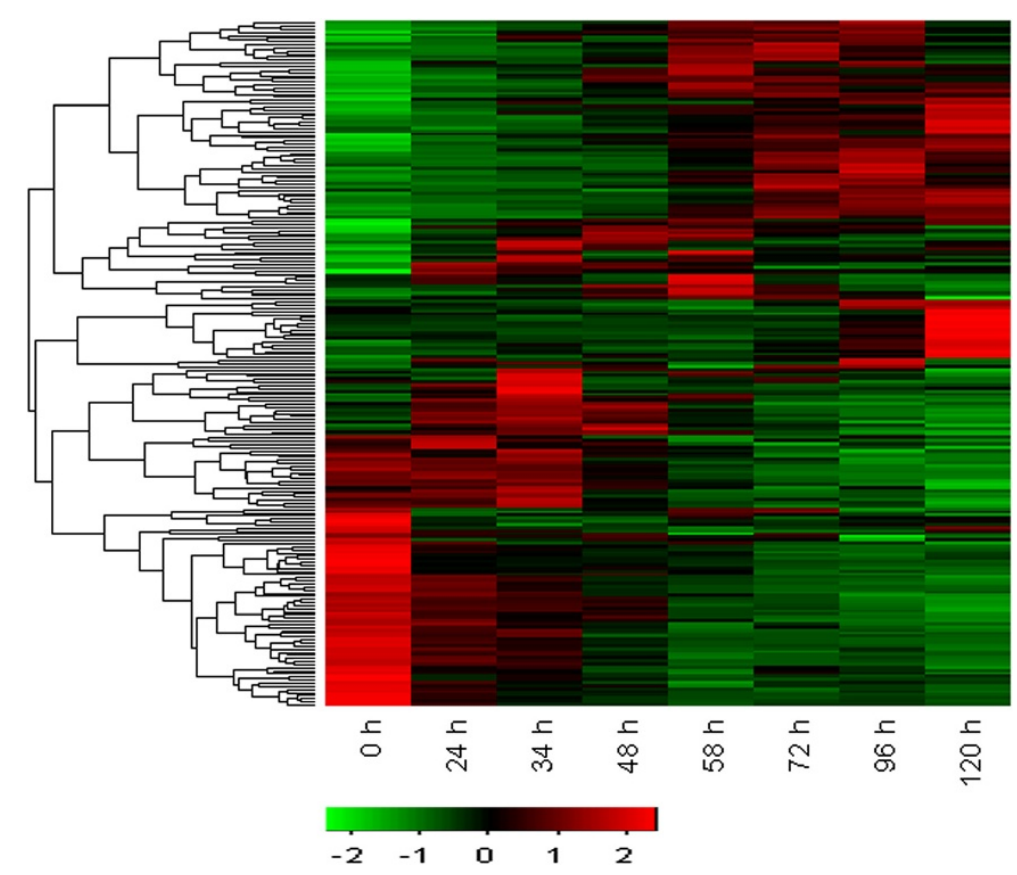

Figure 5 Expression pattern of differentially expressed transcription factors.

unigenes. Results obtained by RT-qPCR were compared to data obtained by RNA-seq (Figure 6). Results indicated that the expression patterns obtained using the two methods were similar (Figure 6), confirming the reliability of the expression data obtained by RNA-seq.

\section{Discussion}

A similar number of DEGs were detected at each time point (Table 1) indicating that the number of DEGs did not dramatically increase at any specific stage of seed hair development. The even distribution of DEGs across all time points thus indicates that seed hair development is a highly regulated process. Few reports, outside of the present study, have examined seed hair development in Populus. The GO enrichment for cell wall and cellulose related descriptors, as well as the corresponding expression data for genes represented by these GO terms, clearly indicate that cellulose is an essential component of poplar seed hairs. In this regard, it is very similar to the component in cotton fibers.

\section{Association between seed and seed hair}

In the present study, a set of morphological observations provided a complete overview of development of poplar seed hairs. Importantly, the placental tissue inside the ovary was confirmed as the initiation site for poplar seed hairs. Floral buds used in this study were not pollinated, so no seeds were produced. Therefore, the origin of poplar seed hair differs from seed hair trichomes, such as cotton fibers, which develop from the epidermal layer of the seed coat outside of the fertilized ovules. Similar to our finding that trichome development could occur without fertilization, Sofia and Rober [22] reported that exogenous application of plant hormones to unfertilized ovules of Gossypium hirsutum, in vitro could result in cotton fiber production. These results indicate that fertilization is not necessarily a prerequisite for seed hair development to occur. Rather, other regulators promoting seed trichome initiation may exist and the activity of these different regulators may result in the differences in the initiation site between cotton and poplar seed hair trichomes. Since seed development and seed hair development are not linked in poplar, the term "ovary fiber" may be more accurate and appropriate for describing the trichomes in poplar.

\section{Cell cycle and cell division}

Nuclei staining deep red with the saffranin dye were observed in the early stages of seed hair development in poplar. Visible nuclei could not be observed until trichome development was initiated (Figure 1A, B, C, D) but were easily discerned in elongating trichome cells at later stages of development (Figure 1F, H, J). The observations of deeper stained nuclei after trichome initiation, along with the single-celled extension of trichome cells, indicate that trichome cells had undergone DNA replication but that cell division did not occur. Similar endoreduplication has been reported in Arabidopsis leaf 

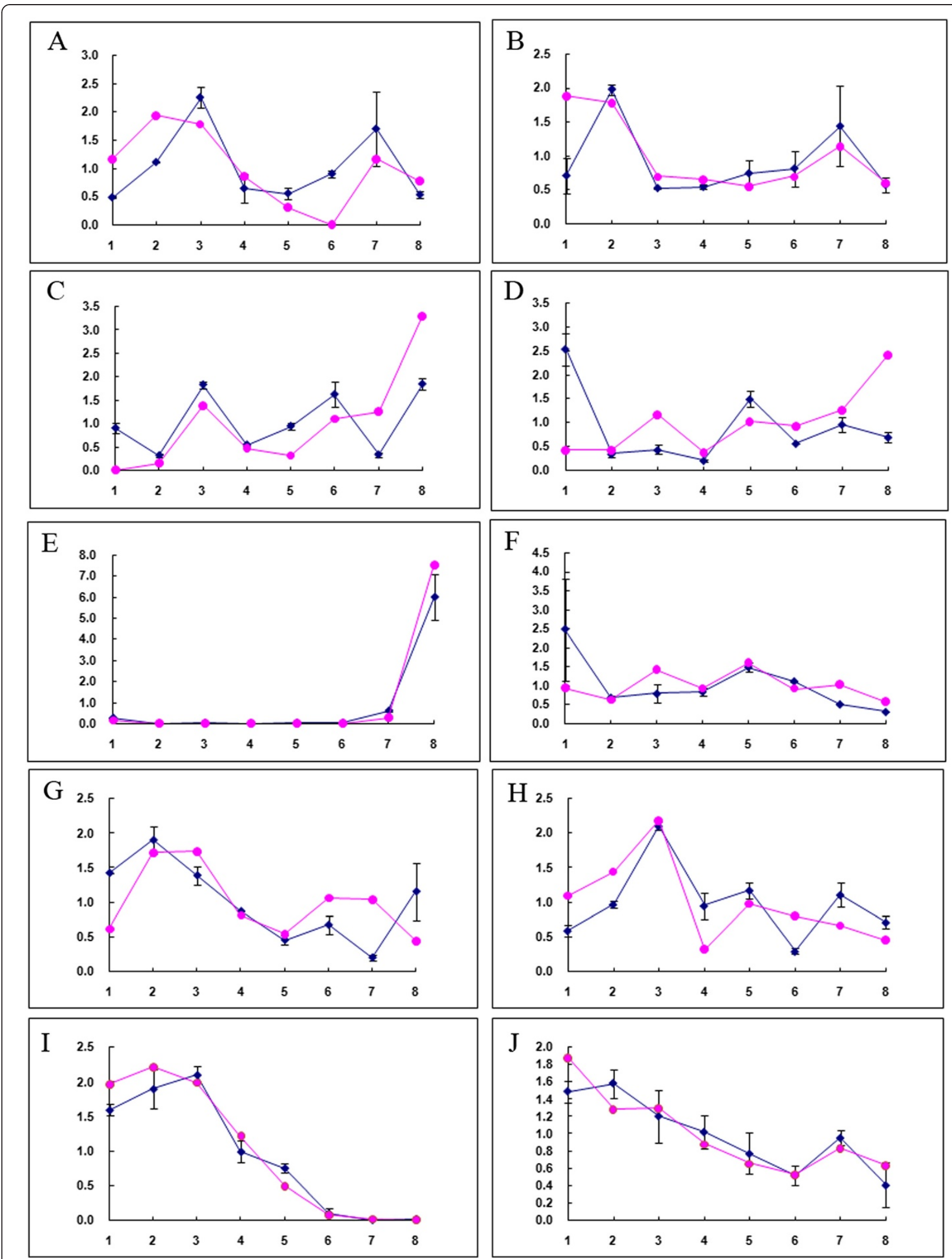

Figure 6 (See legend on next page.) 
(See figure on previous page.)

Figure 6 RT-qPCR confirmation of expression profiles obtained by RNA-seq transcriptome analysis. Fold-change in transcript abundance obtained by both RT-qPCR and RNA-Seq are presented on the same graph for eight different genes. Representative encoding genes are shown in A-J. Lines with magenta color are data obtained by RNA-seq, and blue lines represented the validated data by RT-qPCR. A-J are sucrose-phosphate synthase, trehalose 6- phosphatase, beta-D-xylosidase 4, glucose-1-phosphate adenylyltransferase, polygalacturonase, beta-fructofuranosidase, protein phosphatase $2 \mathrm{C}-1 / \mathrm{PP} 2 \mathrm{C}-1$, protein phosphatase $2 \mathrm{C}-2$ /PP2C-2, YABBY-1, YABBY-2 respectively.

trichomes and elongating cotton fiber cells [23-25]. Hertzberg et al. (2001) identified histone $\mathrm{H} 4$ as a marker for cell division proceeded by DNA replication [26]. In our data, many annotated unigenes having functions similar to histone H4 exhibited a common expression pattern. All of the histone H4-like genes were most abundant at the early stage of poplar seed hair development, and then dramatically decreased at later time points. Four rounds of endoreduplication have been reported to occur during Arabidopsis leaf trichome development [27], however, the exact number of endoreduplication cycles that occur during poplar seed hair development could not be determined in the present study. Comparison of nuclear size in poplar seed hair cells at $58 \mathrm{~h}$ with other common cells, suggested that at least one endoreduplication cycle occurred before this time point. The increased enrichment of GO terms related to DNA replication and pyrimidine base biosynthesis at $72 \mathrm{~h}$, however, indicated a second cycle of endoreduplication.

A SIAMESE (SIM) mutation in Arabidopsis leading to a phenotype with multi-cellular trichomes has been reported [24]. A similar phenotype of multi-cellular trichomes from the over-expression of a B-type cyclin gene was also reported [27]. This suggests an inhibitory role of SIM on B-type cyclin gene expression and that B-type cyclin genes may play an important role during the normal cycle of mitosis. No SIM homologues, however, were identified in this study, however, four B-type cyclin unigenes were all down-regulated in our dataset, right after the initiation of seed hair cells and their expression remained low at all of the subsequent time points. These results suggest that cell division activity may have been repressed since B-type cyclin normally triggers the transition from the G2 stage to mitosis [28]. The absence of SIM gene expression and the expression pattern of B-type cyclin genes observed in the current study were consistent with the model for plant trichome cell differentiation proposed by Hulskamp et al. [5]. In their model, SIM repression of $C Y B$ gene expression and endoreduplication cycles were thought to cut the cell cycle short by skipping the $G 2$ and $M$ phases of the mitotic cycle.

\section{Cell fate determination of poplar seed hairs}

In addition to endoreduplication, previous studies on Arabidopsis and cotton fiber trichome development identified a set of genes regulating trichome formation patterns, trichome branching and the directionality of their growth [8]. Most of the genes reported in Arabidopsis were also identified during the annotation of the assembled sequences acquired in the present study (Table 2), suggesting that poplar seed hair development may share many similarities with Arabidopsis leaf trichome development. GLABROUS 1 (GL1), an important component of the trichome-promoting complex regulating the determination of trichome cell fate, however, was not identified in our dataset. The AtGL1 protein sequence was used to perform a BLAST against the Populus trichocarpa genome, and a significant match was identified, indicating the existence of a GL1 gene in poplar. When sequences obtained in the present study were mapped again to the genome sequence of Populus trichocarpa, expression of poplar homologs of AtGL1 was detected. These data suggest that the inability to detect GL1 gene expression in the current study was most likely due to errors or problems in sequence assembly.

Table 2 Trichome-related genes and their putative Poplar homologs

\begin{tabular}{|c|c|c|c|}
\hline $\begin{array}{l}\text { Arabidopsis gene } \\
\text { symbols }\end{array}$ & $\begin{array}{l}\text { putative homolog } \\
\text { sequence ID }\end{array}$ & e-value & $\begin{array}{l}\text { Differentially } \\
\text { expressed }\end{array}$ \\
\hline$\overline{G L 1}$ & $\mathrm{~N} / \mathrm{A}$ & $\mathrm{N} / \mathrm{A}$ & $\mathrm{N}$ \\
\hline GL2 & comp95865_c1_seq1 & 0.0 & $N$ \\
\hline EGL3 & comp71893_c0_seq1 & $1 e-44$ & Y \\
\hline TTG1 & comp91443_c0_seq1 & 0.0 & $\mathrm{~N}$ \\
\hline TTG2 & comp93187_c0_seq1 & $1 e-109$ & $\mathrm{~N}$ \\
\hline MYB23 & $\mathrm{N} / \mathrm{A}$ & $\mathrm{N} / \mathrm{A}$ & $\mathrm{N}$ \\
\hline TRY & comp81626_c1_seq1 & $2 \mathrm{e}-35$ & $N$ \\
\hline CPC & comp87626_c0_seq1 & $1 e-32$ & $\mathrm{~N}$ \\
\hline ETC1 & $\mathrm{N} / \mathrm{A}$ & N/A & $\mathrm{N}$ \\
\hline HDG11 & comp96395_c1_seq1 & 0.0 & $\mathrm{~N}$ \\
\hline HDG12 & comp82643_c0_seq1 & $2 e-29$ & $\mathrm{~N}$ \\
\hline NOK & comp80738_c0_seq1 & $8 e-45$ & Y \\
\hline PYM & comp94519_c1_seq1 & $6 e-45$ & $N$ \\
\hline HYP6 & comp88392_c0_seq1 & 0.0 & $\mathrm{~N}$ \\
\hline HDG2 & comp95785_c0_seq1 & 0.0 & $\mathrm{~N}$ \\
\hline PEL3 & comp83936_c0_seq1 & 0.0 & Y \\
\hline
\end{tabular}

$\mathrm{N} / \mathrm{A}$, indicates Not Available. E-value was estimated by tBLAST programme of sequences in this study against protein sequences from The Arabidopsis Information Resource (TAIR) (http://www.arabidopsis.org/), N and $\mathrm{Y}$ in last column indicates genes during seed hair development process are stably expressed or differentially expressed. 
MicroRNAs play an important post-transcriptional role, regulating growth, development, flowering, metabolism, and resistance to biotic and abiotic stress. Functional analyses of microRNAs during cotton fiber development have also been recently explored and the miR156 family was found to be up-regulated during cotton development. Among other things, miR156 targets SQUAMOSA PROMOTER BINDING PROTEIN LIKE 9 (SPL9), which defines an endogenous flowering pathway and temporally controls trichome distribution during flower development by binding to promoters of TCL1 and TRY, which are negative regulators of trichome initiation [29]. In the present study, SPL9 exhibited the lowest level of expression among all of the members of the SPL gene family. Thus, the large onset of poplar seed hair development, which coincidently occurs during the annual phase of poplar flowering, may be related to the low expression of SPL9 since this gene has an inhibitory role on trichome formation. It would be interesting to investigate the expression of SPL9 in other floral organs since poplar seed hair formation is a spatially and temporally regulated process.

\section{Comparative study of highly and dynamically expressed genes}

In nature, the highly size-exaggerated cotton fiber cell and the epidermal trichome cells on Arabidiopsis leaf had become important resources for us to perform a comparative study, which may facilitate greatly to the identification of genes important for seed hair growth for poplar. By isolating trichome from shoots and the respective transcriptome sequencing of isolated trichome and processed shoots from Arabidopsis, Mark et. al., [30] had find a majority of co-expressed genes and many trichome-specific genes, comprising 12 transcription factors and 4 other miscellaneous genes (Table 2). Out of these genes, NOK, HDG2 and PEL3 were found upregulated at the late development stage, with at least 5-fold change occurred. Similar to the expression pattern of PEL3 gene, an increasing expression during development process was revealed on a BURP domain-containing protein gene, in soybean which shared a high degree of homology with the cotton GhRDL1 gene [31,32]. The role of the plant-specific BURP gene in seed hair development can be learned from the findings of the expression profile comparison on soybean, in which, the high expression of BURP was discovered in clark standard (CS) wild type soybean, but the weak expression was seen on the clark glabrous/hairless mutant (CG). Besides, many SNPs and indels with this gene were found on the CS isoline [33].

To begin to explore more genes involved in seed hair initiation and the later elongation stage, expression of genes with Arabidopsis BLAST hits were sorted in a descending order. Of the top five most abundant genes, it is interesting to note the late embryogenesis abundant protein (LEA) family gene that showed a steady but a little decreasing expression profile. But another LEA protein unigene had behaved at least 5-7 fold-change of up-regulation during the $24 \mathrm{~h}-48 \mathrm{~h}$ of the development. In previous reports, LEA protein was known to play significant roles as a reactive oxygen species (ROS) scavenger [34]. A proteomic profiling comparison of the developing fiber cell, performed between the wild type and the domesticated Gossypium barbadense, had indicated an improved abundance of ROS scavenging protein to detoxify $\mathrm{H}_{2} \mathrm{O}_{2}$ during the long artificial domestication process [35]. Other ROS homeostasis maintaining genes, such as ascobate peroxidase (APX), phospholipase D alpha (PLD $\alpha)$, cyclophilin, and glutathione S-transferase family protein (GST) are also found dramatically changed in this study [36-38]. It is worth noting that, the significant up-regulation with GST at 48 h-58 h, coupled with the high expression of LEA protein gene during $24 \mathrm{~h}-48 \mathrm{~h}$, might had provided a direct evidence for the promoting role of ROS pathway to the seed hair initiation or the early seed hair trichome development in poplar.

To better improve our understanding of many important biological processes, a further GO analysis of the top 5000 highly expressed genes were performed at level three. Of the total 42 GO slims retrieved for cellular component, 14 were found to be associated with organelle or membrane, e.g., intracellular organelle, membranebounded organelle, organelle membrane. In the top 20 GO term list that possess the most number of genes, besides the 13 forementioned organelle or membrane GO terms, "vesicle" was another functional annotation term that worth further analysis (Table 3). In regard to biological process, we found 494 unigenes associated with "transport" and 80 unigenes annotated with "vesicle-mediated transport". These coupled annotation with the highly expressed unigenes had emphasized the importance of active membrane trafficking or transport in poplar seed hair development. The importance of "membrane bound organelles" and "intrinsic to membrane" terms to the initials and elongation of cotton fibers had been demonstrated in some previous reports [39]. TEM image of mature wildtype Arabidopsis trichomes in another study had discerned structures like vacuolar autophagosomes, Golgi apparatus, plasma membrane fused vesicles and plastids [30]. Observations of these membrane advanced organelle confirmed the vesicle coating and transporting between ER-Golgi traffic, vacuole and trans-Golgi traffic [40-42]. The highly developed traffic of membrane vesicles might accelerate the delivering of membrane or cell wall material to the diffuse growing points of deposition, so as to support the remarkable growth rate of trichome cells and the maintainance of cytoskeleton [43]. 
Table 3 Membrane and organelle related GO terms with cellular component at level 3

\begin{tabular}{llll}
\hline GO Id & GO terms & $\begin{array}{l}\text { Gene } \\
\text { number }\end{array}$ & Percentage (\%) \\
\hline GO:0043229 & Intracellular organelle & 1445 & 28.9 \\
GO:0043227 & Membrane-bounded organelle & 1173 & 23.5 \\
GO:0016020 & Membrane & 772 & 15.4 \\
GO:0044422 & Organelle part & 598 & 12 \\
GO:0044446 & Intracellular organelle part & 594 & 11.9 \\
GO:0044425 & Membrane part & 567 & 11.3 \\
GO:0043228 & Non-membrane-bounded & 406 & 8.1 \\
& organelle & & \\
GO:0031090 & Organelle membrane & 297 & 5.9 \\
GO:0031967 & Organelle envelope & 127 & 2.5 \\
GO:0012505 & Endomembrane system & 113 & 2.3 \\
GO:0043233 & Organelle lumen & 97 & 1.9 \\
GO:0031982 & Vesicle & 46 & 0.9 \\
GO:0031970 & Organelle envelope lumen & 8 & 0.2 \\
\hline
\end{tabular}

\section{Other regulators affecting development of poplar seed hairs}

Trichomes are commonly seen on leaf surfaces, especially on the abaxial side. The asymmetric abaxialadaxial distribution of trichomes on leaves can be seen as a classic feature of leaf polarity. Previous studies indicated that many members of the YABBY gene family were associated with the establishment of polarity $[44,45]$. Aberrations in leaf polarity could result in leaves bearing trichomes on both sides [46]. Aside from research on the abaxial-adaxial polarity of trichomes on Arabidopsis leaves, no other reports exist on the polarity of the distribution of other kinds of trichomes, and little attention has focused on the role of the YABBY family in trichome cell fate or trichome cell development.

The expression of $Y A B B Y 2$ and $Y A B B Y 3$ was reported to be localized to the abaxial region of lateral organs $[47,48]$. The cluster analysis of transcription factors in our current study indicated a high level of expression of two YABBY members from $0 \mathrm{~h}$ to $48 \mathrm{~h}$, the time period during which the cell fate of poplar seed hairs may be determined. After $48 \mathrm{~h}$, a dramatic decrease in expression occurred (Figure 6I, J), with expression of one of these two highly expressed genes being reduced to an extremely low level (Figure 6I). The RT-qPCR analysis validated a higher expression of YABBY members at an early stage of seed hair development and a relative low level of expression at later developmental stages, equivalent to the period of seed hair trichome elongation. The low expression during the latter stages of seed hair development may indicate that YABBY gene expression was not required for elongation of poplar seed hair, however, its high level of expression in early stages of development may have a significant impact on the cell fate of poplar seed hairs. In other words, our results indicate a potential role of YABBY in cell determination of poplar seed hairs but not the establishment of an abaxial-adaxial polarity.

Within the ARF family, the two unigenes with greatest fold-change in expression revealed their increasing upregulation from $48 \mathrm{~h}$ to $96 \mathrm{~h}$, the initiation and elongation stage of seed hair development. Recent studies demonstrated that auxin stimulation can promote the formation of root hairs via the upregulation of Aux/IAA genes $[49,50]$. Thus, the functional role of ARF in auxin response may play a role in stimulating the elongation of seed hairs in poplar.

\section{Conclusion}

Poplar seed hairs are a type of trichome that are produced in such an abundance every spring that they become an environmental annoyance. Little is known about the development of these prolific cells. The results of the current study indicated that the placenta at the base of ovary is the site of seed hair emergence, and that cells targeted to become seed hairs feature an enlarged cell nucleus. A uni-cellular property was maintained during the elongation and subsequent stages of seed hair development. Importantly, a dynamic and comprehensive characterization of the transcriptome of seed hairs during the entire course of seed hair development was obtained using RNA-seq technology. By investigating the dynamics of gene expression, genes potentially involved in trichome cell fate determination, branch formation, and growth directionality were identified. Biologically significant processes, such as endoreduplication and bypassing the normal mitotic cycle, cellulose synthesis facilitating cell wall deposition, as well as the stagespecific regulation of transcription factors were revealed. The morphological observations provided the basis for determining the sampling time used for transcriptome sequencing. Observations of an enlarged nucleus and the filamentous extension of trichomes supported the transcriptome data which indicated or implied that endoreduplication and cellulose synthesis was occurring during seed hair development. The observation of cell cycle and cell division related gene expression, altered by endoreduplication, was suggested to play a role in the maintenance of the single-celled structure of seed hairs. The ability to associate changes in the transcriptome with distinct morphological stages was a valuable tool enabling us to capture and offer a plausible interpretation for the dynamic changes in gene expression and abundance. Poplar seed hairs represent an ideal model for discovering novel mechanisms regulating cell fate determination and cell elongation at the single-cell level. 


\section{Methods}

\section{Plant tissue collection and RNA isolation}

Branches of adult female Populus tomentosa trees were collected in early February, at which time floral buds were developmentally mature. Floral buds or the later catkins were harvested at $0 \mathrm{~h}, 24 \mathrm{~h}, 34 \mathrm{~h}, 48 \mathrm{~h}, 58 \mathrm{~h}$, $72 \mathrm{~h}, 96 \mathrm{~h}$, and $120 \mathrm{~h}$ from the cut branches cultured in clean water at room temperature. Samples were rapidly frozen in liquid nitrogen and then stored at $-76^{\circ} \mathrm{C}$ until further use. Additionally, samples from the eight time points were also fixed with formalin-acetic acid (FAA) fixative solution for morphological and anatomical observation. Total RNA was isolated as described previously [51].

\section{Preparation of paraffin sections}

Dehydration of fixed samples was performed sequentially in $50 \%, 70 \%, 85 \%, 95 \%$, and $100 \%$ ethanol at $2 \mathrm{~h}$ intervals. Tissues were cleared with $1 / 2$ dimethylbenzene $+1 / 2$ ethanol for $2 \mathrm{~h}$, followed by a $2 \mathrm{~h}$-immersion in absolute dimethylbenzene. Wax was infiltrated into tissues at $38^{\circ} \mathrm{C}$ overnight using a mixture of dimethylbenzene and wax dust. Tissues were then immersed in pure liquid wax three times at $4 \mathrm{~h}$ intervals. Tissues, along with melted wax, were placed on a paper box and the wax was rapidly hardened with cold water for subsequent sectioning. Serial sections obtained by using a microtome were pasted onto microscope slides with egg white. The wax was removed and the sections were stained as follows: microscope slides with the sections were immersed in dimethylbenzene for 5 min, $1 / 2$ dimethylbenzene $+1 / 2$ ethanol for $2 \mathrm{~min}, 100 \%$ ethanol for $2 \mathrm{~min}, 95 \%$ ethanol for $2 \mathrm{~min}, 85 \%$ ethanol for $2 \mathrm{~min}, 70 \%$ ethanol for $2 \mathrm{~min}, 50 \%$ ethanol for $2 \mathrm{~min}$, distilled water for $2 \mathrm{~min}, 1 \%$ saffranin for $30 \mathrm{~min}, 50 \%$ ethanol for $2 \mathrm{~min}, 70 \%$ ethanol for $2 \mathrm{~min}$, $85 \%$ ethanol for $2 \mathrm{~min}, 95 \%$ ethanol for $2 \mathrm{~min}, 0.1 \%$ fast green for $1-2 \mathrm{~s}$, acid ethanol for $1-2 \mathrm{~s}, 100 \%$ ethanol for 2 min, $1 / 2$ dimethylbenzene $+1 / 2$ ethanol for $2 \mathrm{~min}$, dimethylbenzene for $5 \mathrm{~min}$. Cover slides were then applied over the sections and the slides were observed with a light microscope.

\section{Processing and assembly of sequence data}

RNAs from eight different developmental stages were sequenced separately using Illumina paired-end technology and an Illumina Hiseq2000 platform. The high throughput sequencing was performed at Beijing Yuanquanyike Biological Technology Co.,Ltd. (Beijing, China). Approximately, 500,000 reads from each raw sequencing dataset were randomly selected for contamination detection, by the method of BLASTN against nucleotide database from the National Center for Biotechnology Information (NCBI) website. Low quality read fragments were removed according to a slicing window method. Fragments greater than 35 bp with an overall quality score above 20 were retained. If two or more segments occurred with the same read, only the longest segment was kept for the next step of assembly. All of the valid reads from the eight datasets were pooled together for de novo assembly, Trinityrnaseq_r2012-06-08, an assembly program based on the de Bruijn graph theory, and developed specially for transcriptome sequence assembly, was used for paired-end assembly with default or optimal parameters. After extract all overlapping $k$-mers, all unique $(k-1)$-mers were sorted and were used to construct transcripts by a greedy algorithm. Parameter $k$ was set as 25 , a value which proved efficient in many transcriptome assemblies, meryl was chosen as the kmer method, the min_contig_length was set as 100, and the group_pairs_distance was set as 500 .

\section{Annotation of unigenes}

Due to the inclusion of potential splicing isoforms in the assembly, the longest transcripts for a certain loci were taken as unigenes. Unigenes were annotated using the publicly available non-redundant protein $(\mathrm{Nr})$ database, with a criterion of similarity above $30 \%$ and e-value above 1e-5. Gene ontology annotation comprising biological process, molecular function, and cellular component was performed using Blast2go [52]. Additionally, KEGG pathway assignments were made using the KAAS server [53].

\section{Estimation of expression abundance}

Reference was made using all assembled transcripts, and all qualified reads from each sequencing dataset were mapped back to reference sequence respectively. Bowtie0.12.7 mapping software was used. Parameters $-\mathrm{v} 3$ and $-\mathrm{a}$-phred64-quals were set allowing for reads mapping to multiple transcripts. After mapping, RPKM was applied to estimate expression abundance due to its ability to eliminate bias from transcript length and sequencing depth. Approximately $77.24 \%$ of all reads were successfully mapped.

\section{Enrichment analysis}

Due to the time-course parameters of this study, seven sets of DEG analysis were separately performed. In each analysis, a criterion of $\mid \log _{2}$ (ratio) $\mid \geq 2$ and probability for fisher test $\leq 0.05$ between the two consecutive time points was used to identify differentially expressed genes. For enrichment analysis of each sampled time point, DEG were set as the foreground and all of the remaining transcripts as the background, Hyper-geometric distribution was employed to detect the significant GO terms and KEGG pathways at a significance level of 0.05 .

\section{Identification of transcription factors}

Unigenes from all assembled sequences were used to perform a BLAST analysis against the transcription 
factors from Populus trichocarpa, available in the Plant Transcription Factor Database (PlantTFDB, http:// planttfdb.cbi.pku.edu.cn/index.php?sp=Pth) $[54,55]$.

\section{Reverse transcription - quantitative PCR (RT-qPCR)}

RQ1 DNase I (Promega) was used to remove contaminating genomic DNA from total RNA. The first -strand cDNA was synthesized using $1 \mu \mathrm{g}$ RNA with oligo (dT) 15 using a reverse transcription system (Promega), subsequently diluted 1:10 with water, and was then used as a template for amplification. Primers utilized are presented in Additional file 2: Table S2. Thermal cycling was performed at $94^{\circ} \mathrm{C}$ for $5 \mathrm{~min}, 94^{\circ} \mathrm{C}$ for $30 \mathrm{~s}, 60^{\circ} \mathrm{C}$ for $20 \mathrm{~s}$, $72^{\circ} \mathrm{C}$ for $30 \mathrm{~s}$, for 35 cycles, then at $72^{\circ} \mathrm{C}$ for $5 \mathrm{~min}$. The Actin8 gene (Genebank ID: EEE71962.1) was selected as internal reference gene. RT-qPCR was performed on a 7500 Fast Real-Time PCR System platform using SYBR Premix Ex Taq kit.

\section{Availability of supporting data}

All sequences were deposited in the National Center for Biotechnology Information (NCBI) and can be accessed in the Short Read Archive (SRA) under accession number: SRP036878. Experiment accession numbers for all 8 distinct development stages are: SRX467610, SRX467612, SRX467616, SRX468207, SRX468238, SRX468240, SRX468242 and SRX468243 respectively.

\section{Additional files}

Additional file 1: Table S1. Gene Ontology enrichment results. Analysis of gene ontology enrichment for all seven sets of differentially expressed genes was performed.

Additional file 2: Table S2. Sequence of primers used in RT-qPCR. Primer sequences in this file were used to detect expression level of genes selected for a further validation of the reliability of RNA-seq technology.

\section{Competing interests}

The authors declared that they had no conflicting interests.

\section{Authors' contribution}

XMA designed the experiment, coordinated and supervised the research. MXY drafted the manuscript. MXY, ZC and XXS had the most contribution to the conduction of RT-qPCR, sequence assembly, sequence annotation and the transcriptome analysis. LXJ had the main responsibility for RNA isolation and assessment of RNA quality of all samples from different stages. JW assisted in the work of sampling floral branches and was in charge of water cultivation of these samples. WHL and HDM had helped improve the manuscript. All authors read and approved the final manuscript.

\section{Acknowledgements}

This work was supported by grants from the Major State Basic Research Development Program (2012CB114505), the Natural Science Foundation of China (31170631), the Changjiang Scholars and Innovative Research Team Program of China (IRT13047), and the National Biological Science Base Foundation of China (J1103516)

\section{Author details}

${ }^{1}$ National Engineering Laboratory for Tree Breeding, Key Laboratory of Genetics and Breeding in Forest Trees and Ornamental Plants, The Tree and Ornamental Plant Breeding and Biotechnology Laboratory, Beijing Forestry University, Beijing 100083, People's Republic of China. ${ }^{2}$ Beijing Center for Physical and Chemical Analysis, Beijing 100094, People's Republic of China.

Received: 13 November 2013 Accepted: 11 June 2014

Published: 14 June 2014

\section{References}

1. Wester K, Digiuni S, Geier F, Timmer J, Fleck C, Hulskamp M: Functional diversity of R3 single-repeat genes in trichome development. Development 2009, 136:1487-1496.

2. Ranger CM, Winter RE, Rottinghaus GE, Backus EA, Ellersieck MR, Johnson DW: Bioactivity of lipophilic metabolites from glandular trichomes of Medicago sativa against the potato leafhopper. J Chem Ecol 2004, 30:1969-1983.

3. Wang G, Tian L, Aziz N, Broun P, Dai X, He J, King A, Zhao PX, Dixon RA: Terpene biosynthesis in glandular trichomes of hop. Plant Physiol 2008, 148:1254-1266.

4. Kang JH, Shi F, Jones AD, Marks MD, Howe GA: Distortion of trichome morphology by the hairless mutation of tomato affects leaf surface chemistry. J Exp Bot 2010, 61:1053-1064.

5. Hulskamp M: Plant trichomes: a model for cell differentiation. Nat Rev Mol Cell Biol 2004, 5:471-480.

6. Hulskamp M, Misra S, Jurgens G: Genetic dissection of trichome cell development in Arabidopsis. Cell 1994, 76:555-566.

7. Larkin JC, Oppenheimer DG, Lloyd AM, Paparozzi ET, Marks MD: Roles of the GLABROUS1 and TRANSPARENT TESTA GLABRA Genes in Arabidopsis Trichome development. Plant Cell 1994, 6:1065-1076.

8. Lee JJ, Woodward AW, Chen ZJ: Gene expression changes and early events in cotton fibre development. Ann Bot 2007, 100:1391-1401.

9. Zhao M, Morohashi K, Hatlestad G, Grotewold E, Lloyd A: The TTG1-bHLHMYB complex controls trichome cell fate and patterning through direct targeting of regulatory loci. Development 2008, 135:1991-1999.

10. Walker AR, Davison PA, Bolognesi-Winfield AC, James CM, Srinivasan N, Blundell TL, Esch JJ, Marks MD, Gray JC: The TRANSPARENT TESTA GLABRA1 locus, which regulates trichome differentiation and anthocyanin biosynthesis in Arabidopsis, encodes a WD40 repeat protein. Plant Cell 1999, 11:1337-1350.

11. Payne $C T$, Zhang F, Lloyd AM: GL3 encodes a bHLH protein that regulates trichome development in arabidopsis through interaction with GL1 and TTG1. Genetics 2000, 156:1349-1362.

12. Zhang F, Gonzalez A, Zhao M, Payne CT, Lloyd A: A network of redundant bHLH proteins functions in all TTG1-dependent pathways of Arabidopsis. Development 2003, 130:4859-4869.

13. Wang S, Chen JG: Arabidopsis transient expression analysis reveals that activation of GLABRA2 may require concurrent binding of GLABRA1 and GLABRA3 to the promoter of GLABRA2. Plant Cell Physio/ 2008, 49:1792-1804.

14. Balkunde R, Pesch M, Hulskamp M: Trichome patterning in Arabidopsis thaliana from genetic to molecular models. Curr Top Dev Biol 2010, 91:299-321.

15. Esch JJ, Chen M, Sanders M, Hillestad M, Ndkium S, Idelkope B, Neizer J, Marks MD: A contradictory GLABRA3 allele helps define gene interactions controlling trichome development in Arabidopsis. Development 2003, 130:5885-5894.

16. Wang HH, Tang RJ, Liu H, Chen HY, Liu JY, Jiang XN, Zhang HX: Chimeric repressor of PtSND2 severely affects wood formation in transgenic Populus. Tree Physiol 2013, 33:878-886.

17. Porth I, Klapste J, Skyba O, Hannemann J, McKown AD, Guy RD, Difazio SP, Muchero W, Ranjan P, Tuskan GA, Friedmann MC, Ehlting J, Cronk QCB, El-Kassaby YA, Douglas CJ, Mansfield SD: Genome-wide association mapping for wood characteristics in Populus identifies an array of candidate single nucleotide polymorphisms. New Phytol 2013, 200:710-726.

18. Guerra FP, Wegrzyn JL, Sykes R, Davis MF, Stanton BJ, Neale DB: Association genetics of chemical wood properties in black poplar (Populus nigra). New Phytol 2013, 197:162-176. 
19. Richet N, Afif D, Huber F, Pollet B, Banvoy J, El Zein R, Lapierre C, Dizengremel P, Perre $\mathrm{P}$, Cabane M: Cellulose and lignin biosynthesis is altered by ozone in wood of hybrid poplar (Populus tremula x alba). J Exp Bot 2011, 62:3575-3586.

20. Zhang B, Su X, Zhou X: A MADS-box gene of Populus deltoides expressed during flower development and in vegetative organs. Tree Physio/ 2008, 28:929-934.

21. Hsu CY, Liu Y, Luthe DS, Yuceer C: Poplar FT2 shortens the juvenile phase and promotes seasonal flowering. Plant Cell 2006, 18:1846-1861.

22. Sofia G, Robert WS: Plant hormones alter fiber initiation in unfertilized, cultured ovules of Gossypium hirsutum. The Journal of Cotton Science 2001, 5:252-258.

23. Taliercio E, Hendrix B, Stewart JM: DNA content and expression of genes related to cell cycling in developing Gossypium hirsutum (Malvaceae) fibers. Am J Bot 2005, 92:1942-1947.

24. Walker JD, Oppenheimer DG, Concienne J, Larkin JC: SIAMESE, a gene controlling the endoreduplication cell cycle in Arabidopsis thaliana trichomes. Development 2000, 127:3931-3940.

25. Van't Hof J: Increased nuclear DNA content in developing cotton fiber cells. Am J Bot 1999, 86:776-779.

26. Hertzberg M, Aspeborg H, Schrader J, Andersson A, Erlandsson R, Blomqvist K, Bhalerao R, Uhlen M, Teeri TT, Lundeberg J, Sundberg B, Nilsson P, Sandberg G: A transcriptional roadmap to wood formation. Proc Natl Acad Sci U S A 2001, 98:14732-14737.

27. Schnittger A, Schobinger U, Stierhof YD, Hulskamp M: Ectopic B-type cyclin expression induces mitotic cycles in endoreduplicating Arabidopsis trichomes. Curr Biol 2002, 12:415-420.

28. Weingartner M, Pelayo HR, Binarova P, Zwerger K, Melikant B, de la Torre $C$, Heberle-Bors E, Bogre L: A plant cyclin B2 is degraded early in mitosis and its ectopic expression shortens G2-phase and alleviates the DNA-damage checkpoint. J Cell Sci 2003, 116:487-498.

29. Yu N, Cai WJ, Wang S, Shan CM, Wang LJ, Chen XY: Temporal control of trichome distribution by microRNA156-targeted SPL genes in Arabidopsis thaliana. Plant Cell 2010, 22:2322-2335.

30. Marks MD, Wenger JP, Gilding E, Jilk R, Dixon RA: Transcriptome analysis of Arabidopsis wild-type and gl3-sst sim trichomes identifies four additional genes required for trichome development. Mol Plant 2009, 2:803-822.

31. Xu H, Li Y, Yan Y, Wang K, Gao Y, Hu Y: Genome-scale identification of soybean BURP domain-containing genes and their expression under stress treatments. BMC Plant Biol 2010, 10:197.

32. Granger C, Coryell V, Khanna A, Keim P, Vodkin L, Shoemaker RC: Identification, structure, and differential expression of members of a BURP domain containing protein family in soybean. Genome 2002, 45:693-701.

33. Hunt M, Kaur N, Stromvik M, Vodkin L: Transcript profiling reveals expression differences in wild-type and glabrous soybean lines. BMC Plant Biol 2011, $11: 145$.

34. Wienkoop S, Zoeller D, Ebert B, Simon-Rosin U, Fisahn J, Glinski M, Weckwerth W Cell-specific protein profiling in Arabidopsis thaliana trichomes: identification of trichome-located proteins involved in sulfur metabolism and detoxification. Phytochemistry 2004, 65:1641-1649.

35. Hu G, Koh J, Yoo MJ, Grupp K, Chen S, Wendel JF: Proteomic profiling of developing cotton fibers from wild and domesticated Gossypium barbadense. New Phytol 2013, 200:570-582.

36. Zou J, Song L, Zhang W, Wang Y, Ruan S, Wu WH: Comparative proteomic analysis of Arabidopsis mature pollen and germinated pollen. J Integr Plant Biol 2009, 51:438-455.

37. Dai S, Chen T, Chong K, Xue Y, Liu S, Wang T: Proteomics identification of differentially expressed proteins associated with pollen germination and tube growth reveals characteristics of germinated Oryza sativa pollen. Mol Cell Proteomics 2007, 6:207-230.

38. Chen Y, Chen T, Shen S, Zheng M, Guo Y, Lin J, Baluska F, Samaj J: Differential display proteomic analysis of Picea meyeri pollen germination and pollen-tube growth after inhibition of actin polymerization by latrunculin B. Plant J 2006, 47:174-195.

39. Taliercio EW, Boykin D: Analysis of gene expression in cotton fiber initials. BMC Plant Biol 2007, 7:22

40. Holthuis JC, Nichols BJ, Pelham HR: The syntaxin Tlg1p mediates trafficking of chitin synthase III to polarized growth sites in yeast. Mol Biol Cell 1998, 9:3383-3397.

41. Sanderfoot AA, Raikhel NV: The specificity of vesicle trafficking: coat proteins and SNAREs. Plant Cell 1999, 11:629-642.
42. Sanderfoot AA, Pilgrim M, Adam L, Raikhel NV: Disruption of individual members of Arabidopsis syntaxin gene families indicates each has essential functions. Plant Cell 2001, 13:659-666.

43. Hovav R, Udall JA, Hovav E, Rapp R, Flagel L, Wendel JF: A majority of cotton genes are expressed in single-celled fiber. Planta 2008, 227:319-329.

44. Bowman JL: The YABBY gene family and abaxial cell fate. Curr Opin Plant Biol 2000, 3:17-22.

45. Siegfried KR, Eshed Y, Baum SF, Otsuga D, Drews GN, Bowman JL: Members of the YABBY gene family specify abaxial cell fate in Arabidopsis. Development 1999, 126:4117-4128.

46. Schwab R, Ossowski S, Riester M, Warthmann N, Weigel D: Highly specific gene silencing by artificial microRNAs in Arabidopsis. Plant Cell 2006, 18:1121-1133.

47. Eshed Y, Izhaki A, Baum SF, Floyd SK, Bowman JL: Asymmetric leaf development and blade expansion in Arabidopsis are mediated by KANADI and YABBY activities. Development 2004, 131:2997-3006.

48. Kumaran MK, Bowman JL, Sundaresan V: YABBY polarity genes mediate the repression of KNOX homeobox genes in Arabidopsis. Plant Cell 2002, $14: 2761-2770$

49. Masucci JD, Schiefelbein JW: The rhd6 mutation of Arabidopsis thaliana alters root-hair initiation through an auxin- and ethylene-associated process. Plant Physiol 1994, 106:1335-1346.

50. Rahman A, Hosokawa S, Oono Y, Amakawa T, Goto N, Tsurumi S: Auxin and ethylene response interactions during Arabidopsis root hair development dissected by auxin influx modulators. Plant Physiol 2002, 130:1908-1917.

51. Chang S, Puryear J, Cairney J: A simple and efficient method for isolating RNA from pine trees. Plant Mol Biol Reptr 1993, 11:113-116.

52. Conesa A, Gotz S: Blast2GO: a comprehensive suite for functional analysis in plant genomics. Int J Plant Genomics 2008, 2008:619832

53. Moriya Y, Itoh M, Okuda S, Yoshizawa AC, Kanehisa M: KAAS: an automatic genome annotation and pathway reconstruction server. Nucleic Acids Res 2007, 35:W182-W185.

54. Zhang H, Jin J, Tang L, Zhao Y, Gu X, Gao G, Luo J: PlantTFDB 2.0: update and improvement of the comprehensive plant transcription factor database. Nucleic Acids Res 2011, 39:D1114-D1117.

55. Guo AY, Chen X, Gao G, Zhang H, Zhu QH, Liu XC, Zhong YF, Gu X, He K, Luo J: PlantTFDB: a comprehensive plant transcription factor database. Nucleic Acids Res 2008, 36:D966-D969.

doi:10.1186/1471-2164-15-475

Cite this article as: Ye et al: Study of seed hair growth in Populus tomentosa, an important character of female floral bud development. BMC Genomics 2014 15:475.

\section{Submit your next manuscript to BioMed Central and take full advantage of:}

- Convenient online submission

- Thorough peer review

- No space constraints or color figure charges

- Immediate publication on acceptance

- Inclusion in PubMed, CAS, Scopus and Google Scholar

- Research which is freely available for redistribution 